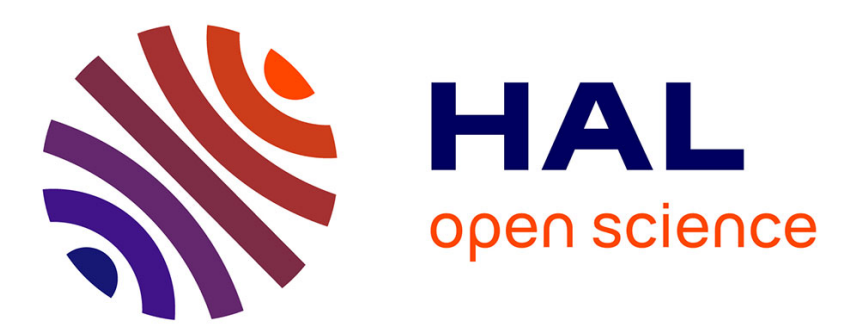

\title{
Performance Study of Co-Located IEEE 802.15.4-TSCH Networks: Interference and Coexistence
}

\author{
Sahar Ben Yaala, Fabrice Theoleyre, Ridha Bouallegue
}

\section{To cite this version:}

Sahar Ben Yaala, Fabrice Theoleyre, Ridha Bouallegue. Performance Study of Co-Located IEEE 802.15.4-TSCH Networks: Interference and Coexistence. 2016 IEEE Symposium on Computers and Communication (ISCC), Jun 2016, Messina, Italy. pp.513-518, 10.1109/ISCC.2016.7543790 . hal02566004

\section{HAL Id: hal-02566004 https://hal.science/hal-02566004}

Submitted on 6 May 2020

HAL is a multi-disciplinary open access archive for the deposit and dissemination of scientific research documents, whether they are published or not. The documents may come from teaching and research institutions in France or abroad, or from public or private research centers.
L'archive ouverte pluridisciplinaire HAL, est destinée au dépôt et à la diffusion de documents scientifiques de niveau recherche, publiés ou non, émanant des établissements d'enseignement et de recherche français ou étrangers, des laboratoires publics ou privés. 


\section{Performance Study of Co-Located IEEE 802.15.4-TSCH Networks: Interference and Coexistence}

\author{
Sahar Ben Yaala \\ Innov'COM Laboratory, Sup'Com \\ University of Carthage, Tunisia \\ National Engineering School of Tunis \\ University of Tunis El Manar \\ email: sahar_by_ingelive.fr
}

\author{
Fabrice Théoleyre \\ ICube Laboratory \\ CNRS / University of Strasbourg, France \\ email: theoleyreeunistra.fr
}

\author{
Ridha Bouallegue \\ Innov'COM Laboratory, Sup'Com \\ University of Carthage, Tunisia \\ National Engineering School of Tunis \\ University of Tunis El Manar \\ email: ridha.boualleguedsupcom.tn
}

\begin{abstract}
With the large deployment of smart and heterogeneous devices, interest of researchers to define new protocols to meet Internet of Things (IoT) requirements is growing. A particular interest was accorded to define a robust MAC layer for wireless sensor networks, in order to reduce interference caused by other co-located networks and applications using the ISM band. This paper gives a comprehensive study of the Time Slotted channel Hopping IEEE802.15.4, part of the 6TiSCH stack and explains how its TDMA approach improves the reliability with performance guarantees. We also investigate analytically and experimentally the impact of the scheduling algorithm on the reliability. Then, we provide an experimental evaluation of colocated WSN using the FiT-IoT LAB testbed and the OpenWSN Stack. Performance analysis of IEEE802.15.4e-TSCH is achieved with a variable number of co-located synchronized or unsynchronized instances. While this standard is robust for lightly loaded networks, new mechanisms have to be proposed when we have too much traffic or too many interfering networks.
\end{abstract}

Index Terms-IEEE802.15.4e-TSCH; interference; dense deployments; co-existence; synchronization; IoT; experiments

\section{INTRODUCTION}

Our society is facing an unprecedented massive usage of numerical applications. The Internet of Things aims at making the environment smarter: smart buildings may comprise hundreds of sensors which report their measure (e.g. temperature, humidity) to a central controller [1]. Smart decisions may then be computed, triggering some actuators (e.g. HVAC).

Body Area Networks (BAN) expect to see similarly a wide adoption. In particular, wearable devices play a key role in eldercare, healthcare or sportswear applications. Smart objects push their measures to a communicating hub, possibly connected to the Internet.

Recently, IEEE802.15.4-TSCH was proposed to set up an industrial wireless sensor network [2]. An FTDMA schedule is adopted, where each transmitter receives an amount of bandwidth during which it is authorized to transmit. The schedule may be constructed to avoid any interfering transmissions so that the transmitters just have to transmit their frames, without any contention resolution mechanism. Besides, TSCH uses slow channel hopping to make the transmissions more reliable and robust to extern interference. $\mathrm{TSCH}$ is consequently able to guarantee a schedule-free collision, and to maximize the sleeping time of each device. This standard is particularly accurate for many M2M applications in low power lossy networks.

Because of the very large variety of applications, we will surely face with very dense deployments of independent networks. Unfortunately, most of the standards use the unlicensed ISM band: these interfering deployments will possibly create many collisions, wasting bandwidth and energy. We have on the contrary to propose cooperative protocols, able to harmoniously and fairly distribute the bandwidth among each other.

While most of the protocols are evaluated individually, their performance in isolation may be very different when many networks interfere. Thus, we have to identify the pathological situations and to predict the performance decrease. Interference among different technologies have been already studied in the past [3], [4]. In particular, a survey presented the different strategies to deal with co-existing networks [5].

To the best of our knowledge, we present here the first paper to study quantitatively the impact of interfering IEEE802.15.4TSCH networks. The channel hopping approach, paired with a deterministic TDMA schedule makes these networks particular: because a node does not sense the medium before its transmission, an interfering node cannot even be detected. We propose consequently to adopt a systematical approach to quantify the impact of co-located networks, and to give insights on the solutions to re-compute the schedules to limit the number of collisions.

The contributions of this paper are threefold:

1) we provide a theoretical analysis to quantify the amount of collisions, depending on the clock drifts and the load pressure. This analysis allows to predict the performance decrease, depending on the number of networks, and the traffic pressure;

2) we experimentally characterize the impact on the reliability when several networks are able to operate synchronously. This represents an optimistic case, where colocated networks are able to cooperate;

3) we experimentally study the impact on performance when several networks are not synchronized and use a random or a static assignment, depicting the classical scenario of different un-cooperating instances.

\section{RELATED WORK AND TECHNICAL BACKGROUND}

Since many technologies use unlicensed bands, co-located networks must share fairly the bandwidth. In particular, Body Area Networks require to have co-existing and interfering single hop networks [5].

\section{A. Multi-Technology Coexistence}

Several papers focused on the co-existence between Wifi and IEEE 802.15.4 (Zigbee). Petrova et al. studied the impact of the packet size, the packet rate and the SINR on the packet losses for co-located IEEE 802.11 and Bluetooth networks [6] Angrisani et al. adopted a similar approach to study the coexistence between IEEE 802.11 and IEEE 8021.5.4 [7]. 


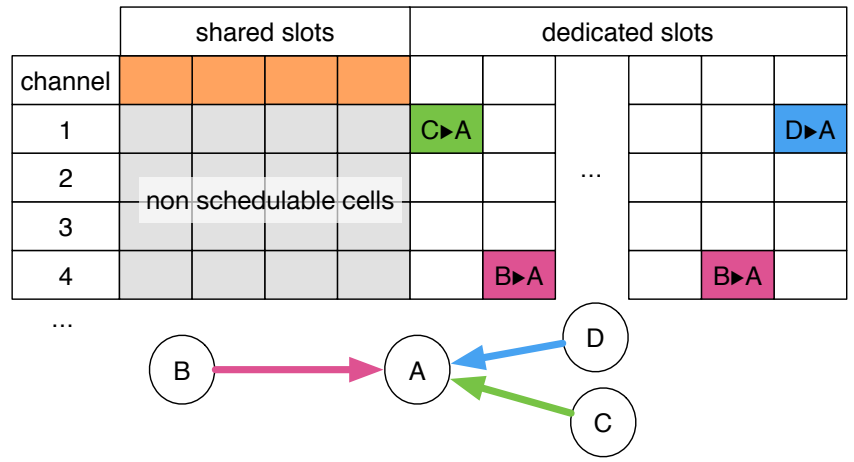

Fig. 1: Schedule in a IEEE802.15.4-TSCH network - illustration with 3 nodes and one border router

Liang et al. [3] studied experimentally the localization of errors when Wifi and Zigbee interfere. They remarked that most colliding bits occur at the beginning of the frame, and propose to use error codes to make the transmissions more reliable.

Hermans et al. classified the source of interference for a IEEE802.15.4 network [4]. The authors proposed a method to identify the source of interference to adopt an accurate countermeasure strategy.

In this paper, we focused rather on the co-existence of multiple networks using the IEEE802.15.4-TSCH standard, which does not use CSMA-CA for all the transmissions.

\section{B. Coexistence of IEEE802.15.4 networks}

While IEEE802.15.4-2006 proposed two different modes, only the beacon-enabled mode enables energy savings in low power lossy networks. In this mode, beacons maintain the synchronization. However, collisions are very frequent, even with one single IEEE802.15.4-2006 instance [8]. Iraqi et al. also evaluated the number of collisions when different IEEE802.15.4-2006 networks use different parameters (superframe duration, and beacon periods) [9].

To reduce this impact, Sun et al. proposed to listen beacons from neighboring BANs and to adapt the schedule to limit the number of collisions [10]. DynMAC proposes to have a central controller which computes a centralized schedule [11]. The authors assume all the instances are owned by the same entity, and that interferences are a priori known. All these solutions assume implicitly the different networks are synchronized, else the different schedule may suffer from clock drifts and may create collisions.

\section{IEEE 802.15.4-TSCH}

IEEE802.15.4e has proposed the TSCH mode for industrial wireless sensor networks [2]. To improve the reliability while maximizing energy savings, the network maintains a schedule, repeated periodically.

TSCH uses a slotframe structure, constituted by a collection of timeslots. The slotframe length is configurable. The obtention of the schedule is out of the scope of the standard.

Since TSCH adopts a TDMA approach, all the nodes must be synchronized. Actually, a node computes the difference between the actual and the expected transmission time of its synchronizing parent. Thus, it can readjust its clock for each transmission, maintaining a sufficiently tight synchronization.
At the beginning of each slot, a node knows if it has to stay awake to receive or transmit a packet or if it may sleep to save its energy. A slot can be either dedicated (without contention) or shared (with a CSMA-CA mechanism to solve the conflicts between the contenders).

To improve the reliability, TSCH proposes to implement a channel hopping scheme. To each transmission opportunity is attached a channel offset. In the TSCH jargon, a cell is a pair of timeslot and channel offset. The channel offset is translated into a frequency to actually use at the beginning of each timeslot:

$$
\text { freq }=(A S N+\text { ch_offset }) \text { mod } 16
$$

where ASN is the absolute sequence number, ch_off $f$ set is the channel offset assigned to this cell in the node's schedule, and mod is the modulo operator. If the number of channels and the slotframe length are mutually prime, we guarantee the same cell will use different frequencies in consecutive slotframes.

Let's consider 3 nodes (B, C, D) which transmit data to the border router (A) (cf. Fig. 1). Some shared slots (in orange) are dedicated for control packets on the channel offset 0 at the beginning of the slotframe. Because of the half duplex condition, all the links must be scheduled during different timeslots since they have a node in common (A). For instance, two cells are reserved for the transmissions from B to A.

While the common schedule avoids the collisions among the nodes inside a given TSCH network, co-located TSCH networks may collide with each other. We propose here to quantify this problem.

\section{6tisch}

6tisch [12] proposes a set of protocols to make the glue between IPv6 (6LoWPAN, CoAP, RPL) and TSCH. It relies on RPL to bootstrap the network, constructing a bidirectional route to the border router (i.e. the sink).

On the Fly Scheduling (OTF) is in charge of monitoring the flows forwarded by a node, and to ask 6top to reserve more cells if the bandwidth is not sufficient. Then, 6top either asks a central controller (the PCE) for a new cell or negotiate directly with the given neighbor.

\section{Theoretical Analysis of the ImpaCt on RELIABILITY WITH A RANDOM SCHEDULING}

We aim here at quantifying analytically the collisions created by colocated networks. We consider the cells are assigned randomly to each device by the Path Computation Element (PCE) to minimize the probability of collision. When having co-located networks, a random strategy is more efficient to avoid repetitive collisions. Since both instances adopt the same strategy, they should avoid assigning the same colliding cells.

For the sake of simplicity, we assume the networks are colocated: any pair of transmitters (from any TSCH instance) collides for sure. We also assume that a collision occurs if the timeslots of the transmitters overlap, even partly, e.g. packets with the largest length are transmitted and fill the timeslot.

We consider the following scenarios:

Synchronized networks represent the most optimistic scenario.

We assume we have an extern source of synchronization, and both TSCH instances use the same slotframe structure (i.e. length, number of shared cells). We may obtain 


\section{TABLE I: Notation}

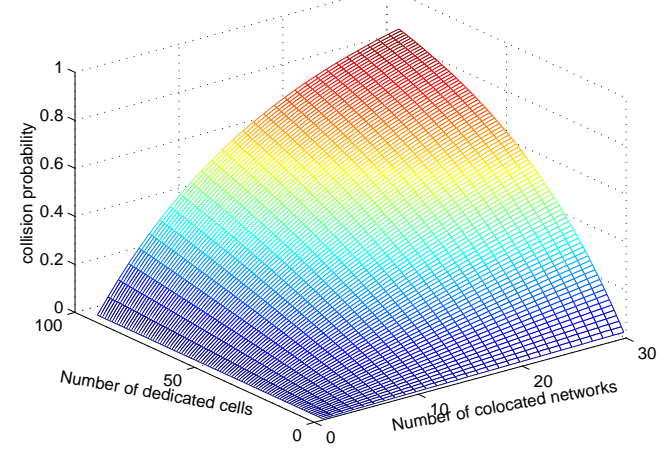

Fig. 2: Collisions among different synchronized instances

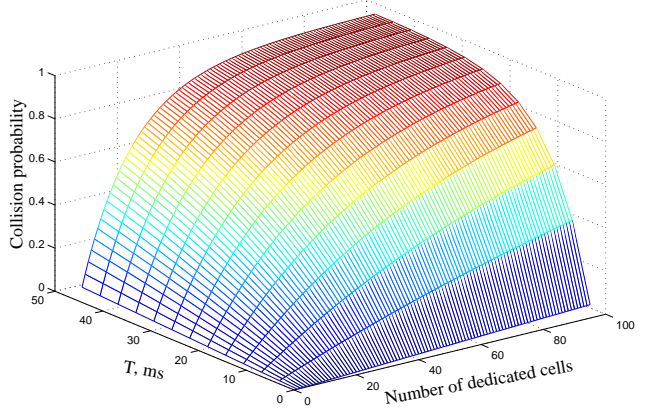

Fig. 3: Collisions among different asynchronous instances

this performance if we are able to introduce cooperation between these instances (i.e. implicit or explicit resynchronization);

Asynchronous networks represent the most common scenario, where several instances are colocated but don't cooperate with each other. They have not the same ASN, and present clock drifts between the different instances. In particular, some cells may collide only in some of the slotframes (depending on their clock drifts).

\section{A. Synchronized Instances}

Let's consider two TSCH networks are co-located but they are synchronized. This means that if they use the same cells, they will collide in every slotframe.

We consider a set $\mathcal{S}_{\text {coloc }}$ of colocated TSCH instances (cf. Table. I for the notation). The instance $i$ has a set $\mathcal{C}(i)$ of dedicated cells allocated for its unicast transmissions.

We focus on the collisions among dedicated cells. Let's consider a cell $c$ randomly chosen. Because the cells are assigned randomly, the collisions may occur during the dedicated part (shared cells have not to be considered). Thus, the probability that the instance $i$ chose it for any of its unicast transmissions is:

$$
\operatorname{Psel}_{\text {sync }}(i)=\frac{|\mathcal{C}(i)|}{\left(\text { SLength }-N_{\text {shared }}\right) * N_{\text {choffset }}}
$$

Let's now compute the collision probability. We consider the TSCH instance $i$. The probability that one of its cells was also chosen by any interfering instance is consequently:

$$
\operatorname{Pcoll}_{\text {sync }}(i)=1-\prod_{k \in \mathcal{S}_{\text {coloc }}-\{i\}}\left(1-\operatorname{Psel}_{\text {sync }}(k)\right)
$$

Finally the number of wasted cells for the instance $i$ is:

$$
\operatorname{Loss}_{\text {sync }}(i)=\operatorname{Pcoll}_{\text {sync }} *|\mathcal{C}(i)|
$$

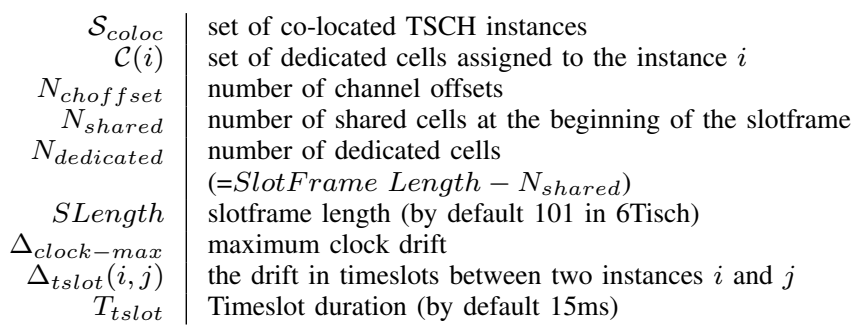

This waste represents actually the amount of energy wasted by collisions (and the loss of radio spectrum).

a) Numerical Results: In Figure 2, we evaluated analytically the impact of dense deployment and duty cycle on the network performance. We can remark that if the number of networks exceeds 15 and we use more then 50 dedicated cells, half of the packets will be lost. Thus, collisions and interference may arise mainly among very dense deployments and for large duty cycle ratio.

\section{B. Asynchronous Instances}

We now consider the case of several TSCH instances, which behave independently, i.e. not synchronized. Since the different instances derive in time, the collisions are time-variant. If initially two cells from two different instances are not sufficiently separated, the clock drifts may be sufficient to provoke a collision.

Let's denote by $\Delta_{\text {clock-max }}$ the maximum clock drift per second. Two instances derive after the time $T$ by:

$$
T * \Delta_{\text {clock-max }}
$$

The crystal oscillator of MSP430 motes presents a typical accuracy of $30 \mathrm{ppm}$ [13]. Thus, $\Delta_{\text {clock }}$ is at most $30 \mu \mathrm{s}$ The actual time difference between two instances will be comprised after e.g. 10 minutes between $-36 \mathrm{~ms}$ and $+36 \mathrm{~ms}$ (i.e. approx. 2 timeslots by default). The probability of collision compared to the synchronous case (eq. 3) must consequently take into account this clock drift.

Without loss of generality, we fix now the reference clock as the clock of the first TSCH instance. The instance $i$ after the time $T$ has a timeslot difference compared to the reference clock:

$$
\Delta_{\text {tslot }}(i, T) \leq 1+\left\lfloor\operatorname{Min}\left(N_{\text {dedicated }}, \frac{T * \Delta_{\text {clock-max }}}{T_{\text {tslot }}}\right)\right\rfloor
$$

By definition, $\Delta_{t s l o t}(1, *)=0$

Let's consider a cell $c$ randomly chosen. The probability that this cell collides during the time $T$ for at least one transmission of the instance $i$ depends on the clock drifts. More precisely, it depends on the number of timeslots swept by this clock drift. Besides, since the slotframes are not required to be aligned, a collision may arise with any cell of the slotframe. Thus, the probability that at least one cell of the instance $i$ collides with the cell $c$ is:

$$
\operatorname{Psel}_{\text {async }}(i, T)=1-\left(1-\frac{\Delta_{\text {tslot }}(i, T)}{N_{\text {dedicated }} * N_{\text {choffset }}}\right)^{|\mathcal{C}(i)|}
$$

The collision probability is consequently similar to the eq. 3 and 4, replacing just $\operatorname{Psel}_{\text {sync }}(k)$ by $\operatorname{Psel}_{\text {async }}(k)$. 


\begin{tabular}{r|c|c} 
Common & Slotframe length & 101 \\
& Shared cells & 5 \\
& Timeslot duration & $15 \mathrm{~ms}$ \\
\hline Synchronized & Traffic type & backlogged \\
Networks & Schedule & hard-coded \\
& Bandwidth Assignment & fixed \\
\hline Asynchronous & Traffic type & CBR \\
Networks & Inter packet time & $6 \mathrm{~s}$ \\
& Schedule & random \\
& Bandwidth Assignment & Adaptive
\end{tabular}

TABLE II: Default parameter values

a) Numerical Results: Figures 3 represent the amount of collisions for the asynchronous case. The collision probability (Fig. 3) is clearly larger than in the synchronous case. These collisions have a strong impact on the reliability: many packets are dropped. Worse, packet drops occur in consecutive slotframes (until the clock drift is superior than the timeslot duration). Thus, an application may suffer from temporary blackouts, very prejudicial for many applications.

\section{EXPerimental Setup to Evaluate the Impact of INTERFERENCE}

\section{A. Experimental platform:}

We used for our experiments the FIT-IoT lab platform (https://www.iot-lab.info/), and in particular the Grenoble indoor testbed, where motes are placed in corridors (false ceiling and floor). The testbed comprises motes based on a STM32 (ARM Cortex M3) micro-controller (ST2M32F103REY). Each mote embeds a AT86RF231 radio chipset, offering an IEEE 802.15.4 compliant PHY layer.

We used the openWSN (https://openwsn.atlassian.net/) implementation of the 6tisch stack. It provides an open-source implementation of IEEE802.15.4e-TSCH, 6top, otf, 6LoWPAN, RPL.

\section{B. 6tisch Configuration and Scenarios}

The control packets (Enhanced Beacons, DIO and DAO for RPL, and 6top frames) use the shared cells at the beginning of the slotframe, with contention. In particular, unicast frames are retransmitted when no acknowledgement is received. Besides, the shared cells are lightly loaded: DIO are for instance transmitted every 30 seconds, and do not create a high load pressure during the shared cells.

The sensitive traffic uses rather the dedicated cells. We modified the 6tisch protocols to evaluate two scheduling functions:

Static scheduling: we mimic a saturated network (i.e. a dedicated cell is always filled by a frame). We implement consequently a backlogged traffic, a packet is generated as soon as the previous one is transmitted. We also deactivated the link-layer retransmissions to have a precise measure of the reliability. Finally, we use a hard-coded schedule (computed offline) to evaluate the impact of repetitive collisions, very prejudicial to industrial applications with high reliability requirements.

Dynamic scheduling: each node generates a CBR traffic, with the default value depicted in table II. We use the autonomous distributed version described in [14]. To evaluate the robustness of such approach, we authorize the packets to be retransmitted. OTF is in charge of overprovisionning cells to deal with retransmissions.

We use the default parameters value as depicted in table II

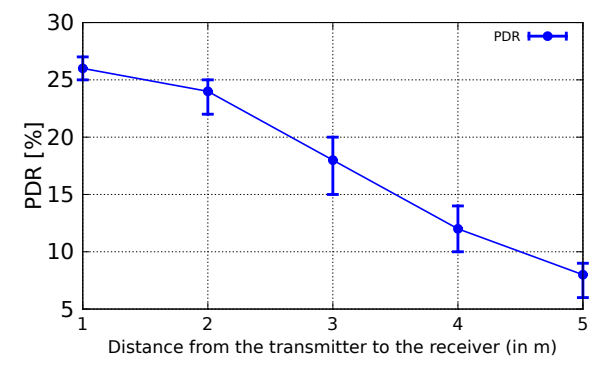

Fig. 4: PDR using synchronized networks with static scheduling - illustration with two interferers separated by 2 meters

\section{Performance criteria}

We instrumented openWSN so that each node reports through its serial port some events. In particular, a node reports when a packet is generated, its destination, its sequence number, etc. We proceed in the same way for each packet received / forwarded by a node. We are consequently able to compute statistics easily, based on all these measures.

We instrumented OpenWSN so that each node reports through its serial port some events. In particular, a node reports when a packet is generated, its destination, its sequence number, etc. We proceed in the same way for each packet received / forwarded by a node. We are consequently able to compute statistics easily, based on all these measures.

We mainly focus on the following metrics of performance:

Packet Delivery Ratio (PDR): ratio of the packets received correctly by the border router and the packets generated by the source.

Number of dedicated cells: we measure for the dynamic scheduling scenario the number of dedicated cells reserved by OTF. A large number of cells means that more retransmissions are sometimes required, reflecting a large number of collisions. Mechanically, the energy consumption is increased and the network capacity is reduced.

We systematically plot the $95 \%$ confidence intervals.

\section{SyNCHRONIZED NETWORKS}

We first mimic several co-located networks which are mutually synchronized (i.e. a single root serves as time reference). We aim here at evaluating the impact of interference when we are able to make the networks cooperate, with an explicit or implicit synchronization: this represents an ideal scenario; All the instances use the same slotframe size, and the shared cells are aligned.

\section{A. Static scheduling - 2 concurrent interfering instances}

We simulate here a scenario where two interfering nodes reserved the same dedicated cells and their instances are synchronized. Since we have backlogged traffic, we aim here at measuring the ability of the system to capture the strongest signal.

We measured first the packet delivery ratio when the source and the sink are progressively separated (Fig. 4). We disabled here the retransmission mechanism to interpret more easily the results. The capture effect allows some packets to be received correctly: when the transmitter is very close to the sink, the number of received packets is maximized, its signal is stronger [15]. When the distance increases, the packet losses also increase. Since the transmissions with the interfering node are synchronized, a large majority of the packets cannot be 


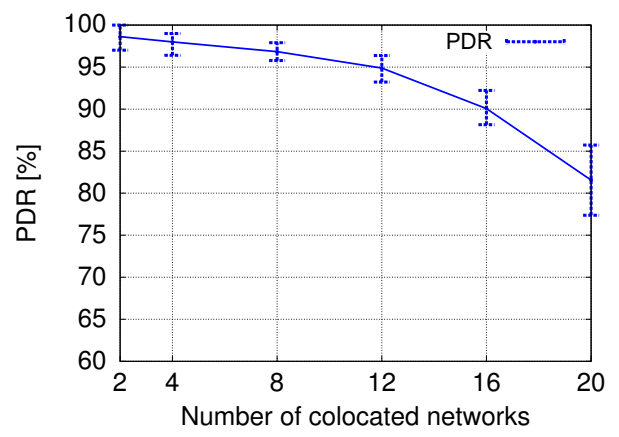

(a) packet delivery ratio

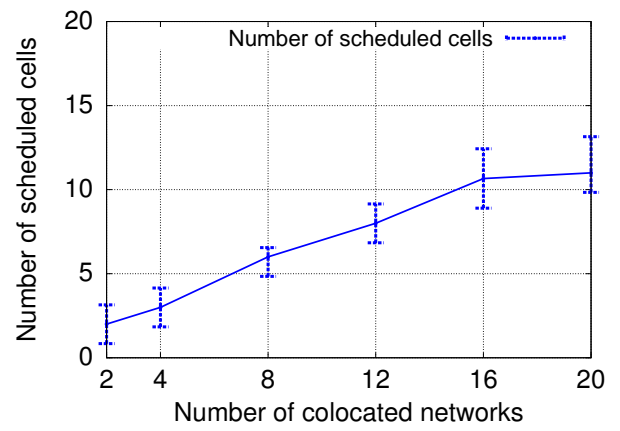

(b) number of allocated cells

Fig. 5: Performance of synchronized networks with random Scheduling - each node is separated by two meters to its sink

decoded by the receiver, even when the transmitter is close to the receiver (i.e. 1 meter).

\section{B. Random Scheduling - Concurrent Instances}

We now measure the packet delivery ratio when we increase the number of TSCH instances, and we let each instance negotiates autonomously the dedicated cells (Fig. 5a)). Retransmissions are here authorized. Even when each instance assigns autonomously the cells, the number of collisions is limited, and the reliability is high. We achieve an almost $100 \%$ delivery ratio even with 4 interfering instances. Unfortunately, the PDR significantly falls when more than 12 nodes are interfering: too many collisions occur, even with 16 different channels. More collisions means also that the packets tend to stay longer in the buffers, triggering the reservations of additional dedicated cells for the retransmissions (Fig. 5b).

In conclusion, random scheduling is efficient only when a limited number of contenders exist. Else, the TSCH network becomes quickly unreliable, unable to fulfill its task.

\section{ASYNCHRONOUS NETWORKS}

We now focus on inter-WSN interference: several IEEE802.15.4e-TSCH networks are deployed and may interfere with each other. They have distinct border routers, and are not synchronized. We aim here at investigating how co-deployments without cooperation may affect the performance of the TSCH schedule.

\section{A. Static Scheduling}

We first simulate dense deployments (e.g. Body Area Networks), and fixed the distance from each transmitter to its border router to 2 meters. We hard-coded the schedule and deactivated the retransmissions to study more accurately the reliability by controlling finely the reserved bandwidth. Since

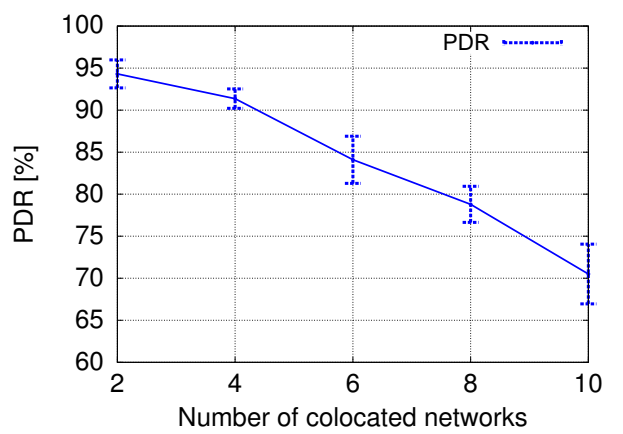

(a) packet delivery ratio

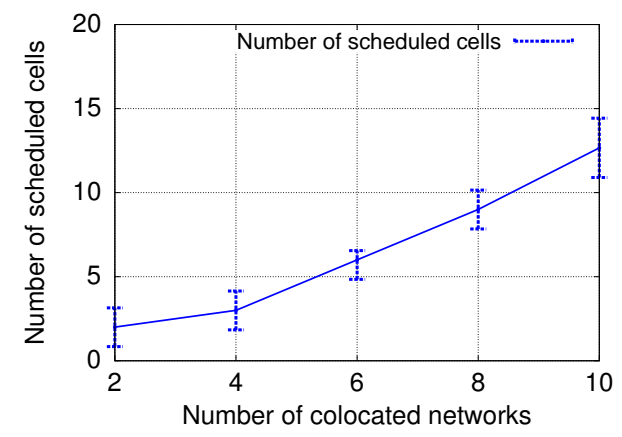

(b) number of allocated cells

Fig. 6: Performance of unsynchronized networks with random Scheduling-distance between co-located networks $=2 \mathrm{~m}$

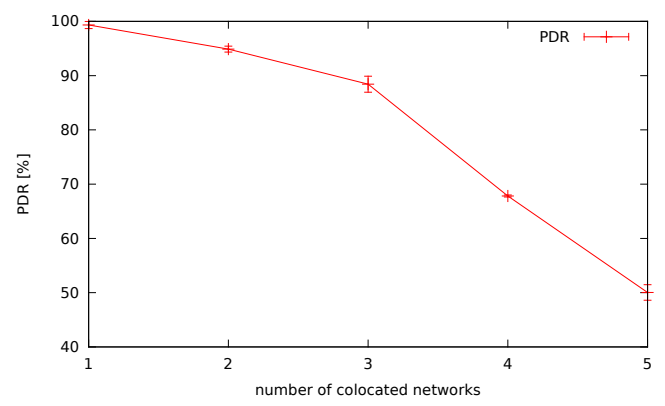

Fig. 7: Performance of unsynchronized networks with static Scheduling-distance between co-located networks $=2 \mathrm{~m}$

the networks are not synchronized, a pair of dedicated may collide in some of the slotframes, depending on the clock drifts.

We first measured the packet delivery ratio (fig. 7). When one interfering network is present, the number of collisions is very reduced, almost $100 \%$ of the packets are correctly delivered. However, the reliability decreases very quickly when more than 2 interfering networks exist. For instance, 4 interfering TSCH instances create many collisions, even if each of them has one unique dedicated cell to transmit data packets. In that case, only $70 \%$ of the packets are delivered correctly. We can verify that the packet delivery ratio is significantly reduced compared to the cooperative scenario where the TSCH instances are synchronized (Fig. 5a). Indeed, the collisions may arise between a pair of interfering networks even if the cells partially overlap. Besides, the number of collisions for a given cell varies over the time. Thus, a dedicated cell may suffer from a temporary blackout because of an interfering network.

In conclusion, dense deployments are only acceptable for very low duty cycles, with a very small quantity of traffic to forward. Else, the collisions become predominant. 


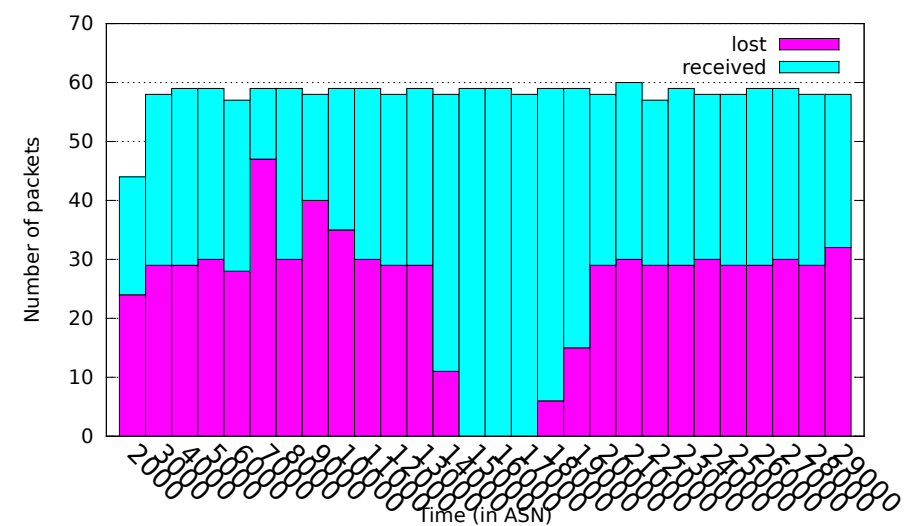

Fig. 8: Loss distribution considering two co-located networks with Random Scheduling - illustration for an experience (15 minutes) with 2 networks of 1 node and 1 sink

\section{B. Random Scheduling}

We mesure in this scenario the performance obtained when several instances operate entirely independently (i.e. not synchronized), and reserve on-demand the dedicated cells autonomously. To limit the impact of the signal strength, all the transmitters are placed 2 meters far from their border router (sink). Figure 6a illustrates the packet delivery ratio: The reliability quickly decreases, and is much lower than when the instances are synchronized with each other. In the same way, the number of cells to reserve is much higher (fig. 6b). With 8 instances, we need on average 6 dedicated cells when the instances are synchronized, and 9 cells $(+50 \%)$ when they operate asynchronously.

We also measured the dynamic of the network, by reporting the packet losses during time intervals along the experiments (fig. 8). We fix the inter packet time to the slotframe length $(1.515 \mathrm{~s})$ and forbid any retransmission to be able to track accurately the packet losses. We can verify that the network spends much time to find an accurate schedule: after an ASN of 14,000, the schedule is now correct and no cell is colliding. Unfortunately, clock drifts quickly make other cells collide, and packets are dropped. In conclusion, adapting the schedule is expensive and useless in unsynchronized TSCH instances.

\section{CONCLUSION}

In this paper, we investigated analytically and experimentally the impact of several co-located instances of IEEE802.15.4eTSCH networks. The collisions may be very prejudicial since they are repetitive: a node doesn't execute any contention resolution mechanism, and all the packets which are transmitted during a colliding cell are lost. If the different networks are able to be synchronized, the collisions are unfrequent. The networks are able to provide a vey high reliability, even when the cells are randomly and autonomously allocated. When the different TSCH networks are not able to be synchronized, the number of collisions is significantly larger. Indeed, clock drifts make the collisions time-variant: one cell may be colliding only during some slotframes. Besides, it is sufficient to have partially overlapping cells to make them collide. As soon as the deployment becomes dense (more than 7 nodes), collisions begin to be predominant. In the future work, we plan to propose mechanisms to detect co-located networks. We aim at proposing a mechanism to re-synchronize either explicitly or implicitly several instances to avoid clock drifts and maintain the performance predictable. We will also propose mechanisms to detect and solve the collisions, without an explicit coordination between the different PCE.

\section{ACKNOWLEDGMENT}

This research was supported by the ICube (University of Strasbourg) project SemSeN .

\section{REFERENCES}

[1] Lars Schor, Philipp Sommer, and Roger Wattenhofer. Towards a zeroconfiguration wireless sensor network architecture for smart buildings. In BuildSys, pages 31-36. ACM, 2009.

[2] IEEE Standard for Local and metropolitan area networks-Part 15.4: LowRate Wireless Personal Area Networks (LR-WPANs) Amendment 1: MAC sublayer. IEEE Std 802.15.4e-2012, 2012.

[3] Chieh-Jan Mike Liang, Nissanka Bodhi Priyantha, Jie Liu, and Andreas Terzis. Surviving wi-fi interference in low power ZigBee networks. In SenSys. ACM, November 2010.

[4] Frederik Hermans, Olof Rensfelt, Thiemo Voigt, Edith Ngai, Lars-Åke Nordén, and Per Gunningberg. Sonic: classifying interference in 802.15 4 sensor networks. In IPSN, pages 55-66. IEEE/ACM, 2013.

[5] Thaier Hayajneh, Ghada Almashaqbeh, Sana Ullah, and Athanasios V Vasilakos. A survey of wireless technologies coexistence in WBAN: analysis and open research issues. Wireless Networks, 20(8):2165-2199, May 2014.

[6] M. Petrova, J. Riihijarvi, P. Mahonen, and S. Labella. Performance study of ieee 802.15.4 using measurements and simulations. In WCNC, volume 1, pages 487-492. IEEE, April 2006.

[7] L. Angrisani, M. Bertocco, D. Fortin, and A. Sona. Experimental study of coexistence issues between ieee $802.11 \mathrm{~b}$ and ieee 802.15 .4 wireless networks. Instrumentation and Measurement, IEEE Transactions on, 57(8):1514-1523, Aug 2008

[8] N. Abdeddaim, F. Theoleyre, M. Heusse, and A. Duda. Adaptive ieee 802.15.4 mac for throughput and energy optimization. In DCOSS, pages 223-230. IEEE, May 2013.

[9] Youssef Iraqi, Tajje-eddine Rachidi, and Peng-Yong Kong. Prevention of collisions among two Wireless Personal Area Networks. In IFIP Wireless and Mobile Networking (WMNC), 2014

[10] Wen Sun, Yu Ge, and Wai-Choong Wong. A lightweight inter-user interference mitigation method in body sensor networks. In WiMob, pages 34-40. IEEE, Oct 2012.

[11] Luiz H A Correia, Thanh-Dien Tran, Vasco N S S Pereira, João C Giacomin, and Jorge M Sá Silva. DynMAC: A resistant MAC protocol to coexistence in wireless sensor networks. Computer Networks, 76(C):1-16, January 2015.

[12] M.R. Palattella, N. Accettura, X. Vilajosana, T. Watteyne, L.A. Grieco, G. Boggia, and M. Dohler. Standardized protocol stack for the internet of (important) things. IEEE Communications Surveys Tutorials, 15(3):1389_ 1406, Third 2013.

[13] Tengfei Chang, Thomas Watteyne, Kris Pister, and Qin Wang. Adaptive synchronization in multi-hop $\{\mathrm{TSCH}\}$ networks. Computer Networks, $76: 165-176,2015$.

[14] Fabrice Theoleyre. A distributed version of 6tisch for high reliability in self-configured low power lossy networks. In submitted, https://clarinet. u-strasbg. fr/ $\sim$ theoleyre/tmp/net16-6tisch-distrib.pdf, 2016.

[15] C. Gezer, C. Buratti, and R. Verdone. Capture effect in ieee 802.15.4 networks: Modelling and experimentation. In ISWPC, pages 204-209. IEEE, May 2010. 